\title{
AS-REGULARITY OF GEOMETRIC ALGEBRAS OF PLANE CUBIC CURVES
}

\author{
AYAKO ITABA $\mathbb{D}$ and MASAKI MATSUNO $\mathbb{D}$ \\ (Received 19 November 2019; accepted 17 February 2021; first published online 22 June 2021)
}

\author{
Communicated by Daniel Chan
}

\begin{abstract}
In noncommutative algebraic geometry an Artin-Schelter regular (AS-regular) algebra is one of the main interests, and every three-dimensional quadratic AS-regular algebra is a geometric algebra, introduced by Mori, whose point scheme is either $\mathbb{P}^{2}$ or a cubic curve in $\mathbb{P}^{2}$ by Artin et al. ['Some algebras associated to automorphisms of elliptic curves', in: The Grothendieck Festschrift, Vol. 1, Progress in Mathematics, 86 (Birkhäuser, Basel, 1990), 33-85]. In the preceding paper by the authors Itaba and Matsuno ['Defining relations of 3-dimensional quadratic AS-regular algebras', Math. J. Okayama Univ. 63 (2021), 61-86], we determined all possible defining relations for these geometric algebras. However, we did not check their AS-regularity. In this paper, by using twisted superpotentials and twists of superpotentials in the Mori-Smith sense, we check the AS-regularity of geometric algebras whose point schemes are not elliptic curves. For geometric algebras whose point schemes are elliptic curves, we give a simple condition for three-dimensional quadratic AS-regular algebras. As an application, we show that every three-dimensional quadratic AS-regular algebra is graded Morita equivalent to a Calabi-Yau AS-regular algebra.
\end{abstract}

2020 Mathematics subject classification: primary 16W50; secondary 16S37, 16D90, 16E65.

Keywords and phrases: AS-regular algebras, Calabi-Yau algebras, elliptic curves, geometric algebras, Koszul algebras, superpotentials.

\section{Introduction}

In noncommutative algebraic geometry, an Artin-Schelter regular (AS-regular) algebra, introduced by Artin and Schelter [1], is one of the main interests. Artin et al. [3] proved that there exists a one-to-one correspondence between three-dimensional AS-regular algebras and regular geometric pairs. This work convinced us that algebraic geometry is very useful for studying even noncommutative algebras. Dubois-Violette [6] and Bocklandt et al. [5] showed that every three-dimensional quadratic AS-regular

The first author was supported by Grants-in-Aid for Young Scientific Research 18K13397 Japan Society for the Promotion of Science.

(C) Australian Mathematical Publishing Association Inc. 2021. This is an Open Access article, distributed under the terms of the Creative Commons Attribution licence (http://creativecommons.org/ licenses/by/4.0/), which permits unrestricted re-use, distribution, and reproduction in any medium, provided the original work is properly cited. 
algebra $A$ is isomorphic to a derivation-quotient algebra $\mathcal{D}(w)$ of a twisted superpotential $w$, and Mori and Smith [13] showed that such $w$ is unique up to nonzero scalar multiples. So it is interesting to study AS-regular algebras using both algebraic geometry and twisted superpotentials. In fact, Mori and Smith [14] classified three-dimensional quadratic Calabi-Yau AS-regular algebras by using superpotentials.

In our previous paper [10], in terms of geometric algebras defined by Mori [12], we determined all possible defining relations for geometric algebras whose point schemes are either $\mathbb{P}^{2}$ or cubic curves in $\mathbb{P}^{2}$ and classified them up to graded algebra isomorphism and up to graded Morita equivalence. However, in [10], we did not check the AS-regularity of these classified geometric algebras. So, one of the aims of this paper is to check their AS-regularity. Note that Iyudu and Shkarin [11] recently gave a list of defining relations of three-dimensional AS-regular algebras by using twisted superpotentials, but gave no proof of AS-regularity of these algebras. For geometric algebras listed in [10, Theorem 3.1], we give a list of candidates of twisted superpotentials to serve our purposes (see Proposition 3.1). By using this list, we give a complete list of superpotentials whose derivation-quotient algebras are three-dimensional quadratic Calabi-Yau AS-regular algebras whose point schemes are not elliptic curves (see Theorem 3.3). By using a twist of a superpotential (in the sense of [13]), we show that the potentials listed in Proposition 3.1 are in fact twisted superpotentials and their derivation-quotient algebras are three-dimensional quadratic AS-regular algebras (see Theorems 3.4 and 3.5). For a geometric algebra $A$ whose point scheme is an elliptic curve in $\mathbb{P}^{2}$, we give a simple condition that $A$ is AS-regular (see Theorem 4.3). As an application of Corollary 3.7 and Theorem 4.3, we prove the following theorem (see Theorem 4.4).

THEOREM 1.1. For every three-dimensional quadratic AS-regular algebra A, there exists a Calabi-Yau AS-regular algebra $S$ such that $A$ and $S$ are graded Morita equivalent.

Theorem 1.1 tells us that, for a three-dimensional quadratic AS-regular algebra $A$, the study of the noncommutative projective scheme $\operatorname{Proj}_{\mathrm{nc}} A$ of $A$ in the sense of Artin and Zhang [2] is reduced to the study of $\operatorname{Proj}_{\mathrm{nc}} S$ for the Calabi-Yau AS-regular algebra $S$. Note that [17, Example 14] gave one example of a three-dimensional cubic AS-regular algebra which is not graded Morita equivalent to any Calabi-Yau AS-regular algebra.

This paper is organized as follows. In Section 2 we recall the definition of an AS-regular algebra defined by Artin and Schelter [1], a Calabi-Yau algebra by Ginzburg [8], a twisted superpotential and a twist of a superpotential in the sense of [13]. We also recall Zhang's twist and twisted algebras from [19] and some lemmas that are needed to show our Theorem 1.1. Moreover, we recall the definitions of a geometric algebra for quadratic algebras introduced by Mori [12], and the result of our previous paper [10]. In Section 3 we prove Theorem 1.1 for geometric algebras whose point schemes are not elliptic curves. Finally, in Section 4, we prove Theorem 1.1 for geometric algebras whose point schemes are elliptic curves in $\mathbb{P}^{2}$. 


\section{Preliminaries}

Throughout this paper, let $k$ be an algebraically closed field of characteristic 0 . A graded $k$-algebra means an $\mathbb{N}$-graded algebra $A=\bigoplus_{i \in \mathbb{N}} A_{i}$. A connected graded $k$-algebra $A$ is a graded $k$-algebra such that $A_{0}=k$. We denote by $\operatorname{GrMod} A$ the category of graded right $A$-modules and graded right $A$-module homomorphisms and we say that two graded $k$-algebras $A$ and $B$ are graded Morita equivalent if the two categories $\operatorname{GrMod} A$ and $\operatorname{GrMod} B$ are equivalent.

2.1. AS-regular algebras and Calabi-Yau algebras. Let $A$ be a connected graded $k$-algebra finitely generated by elements of positive degree. We recall that

$$
\operatorname{GKdim} A:=\inf \left\{\alpha \in \mathbb{R} \mid \operatorname{dim}_{k}\left(\sum_{i=0}^{n} A_{i}\right) \leq n^{\alpha} \text { for all } n \gg 0\right\}
$$

is called the Gelfand-Kirillov dimension of A.

DEFinition 2.1 [1, page 171]. A connected graded $k$-algebra $A$ is called a $d$-dimensional Artin-Schelter regular (AS-regular) algebra if $A$ satisfies the following conditions:

(i) $\operatorname{gldim} A=d<\infty$;

(ii) $\operatorname{GKdim} A<\infty$;

(iii) (Gorenstein condition) $\operatorname{Ext}_{A}^{i}(k, A) \cong \begin{cases}k & \text { if } i=d \text {, } \\ 0 & \text { if } i \neq d .\end{cases}$

Any three-dimensional AS-regular algebra $A$ finitely generated in degree 1 is a graded algebra isomorphic to an algebra of the form

$$
k\langle x, y, z\rangle /\left(f_{1}, f_{2}, f_{3}\right) \text { (quadratic case) or } k\langle x, y\rangle /\left(g_{1}, g_{2}\right) \text { (cubic case), }
$$

where the $f_{i}$ are homogeneous polynomials of degree 2 and the $g_{i}$ are homogeneous polynomials of degree 3 [1, Theorem 1.5 (i)]. In this paper, we focus on three-dimensional quadratic AS-regular algebras.

Let $V$ be a three-dimensional $k$-vector space and $T(V)$ the tensor algebra of $V$. We choose a basis $\left\{x_{1}, x_{2}, x_{3}\right\}$ of $V$. Also, for an algebra $T(V) /(R)$, we choose a basis $\left\{f_{1}, f_{2}, f_{3}\right\}$ of $R \subset V^{\otimes 2}$. We set $\boldsymbol{x}:=\left(x_{1}, x_{2}, x_{3}\right)^{t}$ and $\boldsymbol{f}:=\left(f_{1}, f_{2}, f_{3}\right)^{t}$, where, for a matrix $N, N^{t}$ means the transpose of $N$. There is a unique $3 \times 3$ matrix $M$ with entries in $V$ such that $f=M x$ (see [1, page 177]). From [3, page 34], $T(V) /(R)$ is called standard if there are bases for $V$ and $R$ such that the entries in $x^{t} M$ are also a basis for $R$.

THEOREM 2.2 [3, Theorem 1]. Let $V$ be a three-dimensional $k$-vector space and $R$ a three-dimensional subspace of $V^{\otimes 2}$. Then $T(V) /(R)$ is a three-dimensional AS-regular algebra if and only if $T(V) /(R)$ is standard and the common zero locus in $\mathbb{P}^{2}$ of the $2 \times 2$ minors of the matrix $M$ in the above is empty.

Here, we recall the definition of a Calabi-Yau algebra introduced by [8]. 
Definition 2.3 [8, Definition 3.2.3]. A $k$-algebra $S$ is called $a d$-dimensional Calabi-Yau algebra if $S$ satisfies the following conditions:

(i) $\operatorname{pd}_{S^{\mathrm{e}}} S=d<\infty$;

(ii) $\operatorname{Ext}_{S^{\mathrm{e}}}^{i}\left(S, S^{\mathrm{e}}\right) \cong\left\{\begin{array}{ll}S & \text { if } i=d, \\ 0 & \text { if } i \neq d\end{array} \quad\right.$ (as right $S^{\mathrm{e}}$-modules),

where $S^{\mathrm{e}}=S^{\mathrm{op}} \otimes_{k} S$ is the enveloping algebra of $S$.

For example, it is known that an $n$th polynomial ring $k\left[x_{1}, x_{2}, \ldots, x_{n}\right]$ is $n$-dimensional Calabi-Yau.

We remark that, for a 3-dimensional quadratic AS-regular algebra $A$, the quadratic dual $A^{!}$of $A$ is a Frobenius algebra by [18, Proposition 5.10]. Hence, we can consider the Nakayama automorphism $v_{A}$ ! of $A$ ! . By using the following consequence proved by Reyes et al. [16], we can determine whether these algebras $A$ are Calabi-Yau algebras or not.

LEMMA 2.4 [16, comments after the proof of Example 1.4]. Let $A$ be $a$ three-dimensional quadratic AS-regular algebra. Then A is Calabi-Yau if and only if the Nakayama automorphism $v_{A^{!}}$of $A^{!}$is the identity (that is, $A^{!}$is symmetric).

2.2. Twisted algebras. In this subsection we recall the notions of twisting system and twisted algebra introduced by Zhang [19].

A set of graded $k$-linear automorphisms of $A$, say $\theta=\left\{\theta_{i} \mid i \in \mathbb{N}\right\}$, is called a twisting system of $A$ if $\theta_{n}\left(a \theta_{l}(b)\right)=\theta_{n}(a) \theta_{n+l}(b)$ for all $l, m, n \in \mathbb{N}$ and all $a \in A_{l}, b \in A_{m}$ [19, Definition 2.1]. Let $\theta=\left\{\theta_{i} \mid i \in \mathbb{N}\right\}$ be a twisting system of $A$. Then a new graded and associative multiplication $*$ on the underlying graded $k$-vector space $\bigoplus_{i \in \mathbb{N}} A_{i}$ is defined by

$$
a * b:=a \theta_{l}(b) \text { for all } a \in A_{l}, b \in A_{m} .
$$

We denote by $1_{\theta}$ the identity with respect to $*$. The graded $k$-algebra $\left(\bigoplus_{i \in \mathbb{N}} A_{i}, *, 1_{\theta}\right)$ is called the twisted algebra of $A$ by $\theta$ and is denoted by $A^{\theta}$ [19, Definition 2.3]. Any graded algebra automorphism $\theta \in$ Aut $A$ defines a twisting system of $A$ by $\left\{\theta^{i}\right\}_{i \in \mathbb{N}}$. The twisted algebra of $A$ by this twisting system is denoted by $A^{\theta}$ instead of $A^{\left\{\theta^{i}\right\}_{i \in \mathbb{N}}}$ that is called the twist of $A$ by $\theta$.

LEMMA 2.5 [19, Theorem 3.5]. Let $A$ and $A^{\prime}$ be two connected graded $k$-algebras with $A_{1} \neq 0$. Then $A^{\prime}$ is isomorphic to a twisted algebra of $A$ if and only if GrMod $A$ and GrMod $A^{\prime}$ are equivalent.

LEMMA 2.6 [19, Theorem 5.11 (b)]. Let A be a connected graded k-algebra and $\theta$ be a twisting system of $A$. Then $A$ is a three-dimensional quadratic AS-regular algebra if and only if the twist $A^{\theta}$ of $A$ by $\theta$ is also a three-dimensional quadratic AS-regular algebra. 
2.3. Derivation-quotient algebras. We now recall the definitions of superpotentials, twisted superpotentials and derivation-quotient algebras from [5]. Also, we recall the definition of a twist of a superpotential due to [13] (see Definition 2.9).

Fix a basis $\left\{x_{1}, x_{2}, x_{3}\right\}$ for $V$. For $w \in V^{\otimes 3}$, there exist unique $w_{i} \in V^{\otimes 2}$ such that $w=\sum_{i=1}^{3} x_{i} \otimes w_{i}$. Then the partial derivative of $w$ with respect to $x_{i}(i=1,2,3)$ is $\partial_{x_{i}}(w):=w_{i}$, and the derivation-quotient algebra of $w$ is

$$
\mathcal{D}(w):=T(V) /\left(\partial_{x_{1}} w, \partial_{x_{2}} w, \partial_{x_{3}} w\right)
$$

Note that we call an element $w \in V^{\otimes 3}$ a potential in this paper. We define the $k$-linear $\operatorname{map} \varphi: V^{\otimes 3} \longrightarrow V^{\otimes 3}$ by $\varphi\left(v_{1} \otimes v_{2} \otimes v_{3}\right):=v_{3} \otimes v_{1} \otimes v_{2}$. We write GL( $(V)$ for the general linear group of $V$.

Definition 2.7 [5, Introduction], [13, Definition 2.5]. Let $w$ be a potential in $V^{\otimes 3}$.

(1) If $\varphi(w)=w$, then $w$ is called a superpotential.

(2) If there exists $\theta \in \mathrm{GL}(V)$ such that $(\theta \otimes \mathrm{id} \otimes \mathrm{id}) \varphi(w)=w$, then $w$ is called a twisted superpotential.

REMARK 2.8. By Dubois-Violette [6] and Bocklandt et al. [5], every three-dimensional quadratic AS-regular algebra $A$ is isomorphic to a derivation-quotient algebra $\mathcal{D}(w)$ of a twisted superpotential $w$ (see [6, Theorem 5] and [5, Theorem 6.8]), and by Mori and Smith [13], such $w$ is unique up to nonzero scalar multiples (see [13, Proposition 2.12]).

Definition 2.9 [13, page 390]. For a superpotential $w \in V^{\otimes 3}$ and $\theta \in \operatorname{GL}(V)$,

$$
w^{\theta}:=\left(\theta^{2} \otimes \theta \otimes \mathrm{id}\right)(w)
$$

is called a Mori-Smith twist (MS twist) of $w$ by $\theta$.

For a potential $w \in V^{\otimes 3}$, we set

$$
\operatorname{Aut}(w):=\left\{\theta \in \operatorname{GL}(V) \mid\left(\theta^{\otimes 3}\right)(w)=\lambda w, \exists \lambda \in k \backslash\{0\}\right\} .
$$

For a potential $w \in V^{\otimes 3}$, it follows from [13, Lemma 3.1] that Aut (w) is a subset of Aut $\mathcal{D}(w)$.

Lemma 2.10 [13, Proposition 5.2]. For a superpotential $w \in V^{\otimes 3}$ and $\theta \in \operatorname{Aut}(w)$, we have that $\mathcal{D}\left(w^{\theta}\right) \cong \mathcal{D}(w)^{\theta}$.

LEMMA 2.11. If $w \in V^{\otimes 3}$ is a superpotential and $\theta \in$ Aut (w), then the MS twist $w^{\theta}$ of a superpotential $w$ by $\theta$ is a twisted superpotential.

Proof. Let $w \in V^{\otimes 3}$ be a superpotential and $\theta \in \operatorname{Aut}(w)$. By definition, there exists $\lambda \in k \backslash\{0\}$ such that $\left(\theta^{\otimes 3}\right)(w)=\lambda w$. We set $\theta^{\prime}:=\lambda^{-1} \theta^{3}$ in $\operatorname{GL}(V)$. Since $w$ is a 
superpotential,

$$
\begin{aligned}
\left(\theta^{\prime} \otimes \mathrm{id} \otimes \mathrm{id}\right)\left(\varphi\left(w^{\theta}\right)\right) & =\left(\theta^{\prime} \otimes \mathrm{id} \otimes \mathrm{id}\right)\left(\mathrm{id} \otimes \theta^{2} \otimes \theta\right)(\varphi(w)) \\
& =\lambda^{-1}\left(\theta^{3} \otimes \theta^{2} \otimes \theta\right)(w)=\lambda^{-1}\left(\theta^{2} \otimes \theta \otimes \mathrm{id}\right)(\lambda w)=w^{\theta},
\end{aligned}
$$

so the MS twist $w^{\theta}$ is a twisted superpotential.

REMARK 2.12. If $\theta \in \mathrm{GL}(V) \backslash \operatorname{Aut}(w)$, then the MS twist $w^{\theta}$ of a superpotential $w \in$ $V^{\otimes 3}$ by $\theta$ need not be a twisted superpotential. Indeed, let $w:=x^{3} \in V^{\otimes 3}$. Since $\varphi(w)=$ $w$, we see that $w$ is a superpotential. Take

$$
\theta:=\left(\begin{array}{lll}
0 & 1 & 0 \\
1 & 0 & 0 \\
0 & 0 & 1
\end{array}\right)
$$

in $\mathrm{GL}_{3}(k)$. Then $w^{\theta}=\left(\theta^{2} \otimes \theta \otimes \mathrm{id}\right)(w)=x y x$. Since, for any $\theta^{\prime} \in \mathrm{GL}(V),\left(\theta^{\prime} \otimes \mathrm{id} \otimes\right.$ id) $\left(\varphi\left(w^{\theta}\right)\right)=\theta^{\prime}(x) x y \neq w^{\theta}$, the MS twist $w^{\theta}$ is not a twisted superpotential. Note that we have $\theta \notin \operatorname{Aut}(w)$ by $\left(\theta^{\otimes 3}\right)(w)=y^{3} \neq w$.

DEFINITION 2.13. Let $w \in V^{\otimes 3}$ be a potential.

(1) A potential $w$ is called regular if the derivation-quotient algebra $\mathcal{D}(w)$ is a three-dimensional quadratic AS-regular algebra.

(2) A potential $w$ is called Calabi-Yau if the derivation-quotient algebra $\mathcal{D}(w)$ is a three-dimensional Calabi-Yau AS-regular algebra.

REMARK 2.14. By Bocklandt [4], every three-dimensional quadratic Calabi-Yau AS-regular algebra is isomorphic to a derivation-quotient algebra $\mathcal{D}(w)$ of a superpotential $w$ [4, Theorem 3.1].

Lemma 2.15 [13, Corollary 4.5]. Let $w \in V^{\otimes 3}$ be regular. Then $w$ is Calabi-Yau if and only if it is a superpotential.

LEMMA 2.16. If $w$ is a Calabi-Yau superpotential and $\theta \in \operatorname{Aut}(w)$, then the MS twist $w^{\theta}$ of a superpotential $w$ by $\theta$ is a regular twisted superpotential.

Proof. Let $w \in V^{\otimes 3}$ be a Calabi-Yau superpotential and $\theta \in \operatorname{Aut}(w)$. By Lemma 2.11, the MS twist $w^{\theta}$ of $w$ by $\theta$ is a twisted superpotential, so it is sufficient to show that $w^{\theta}$ is regular. Since $w$ is Calabi-Yau, $\mathcal{D}(w)$ is Calabi-Yau AS-regular, and since $\theta \in \operatorname{Aut}(w)$, by Lemma 2.10 , we have that $\mathcal{D}\left(w^{\theta}\right) \cong \mathcal{D}(w)^{\theta}$. Since it holds from Lemma 2.6 that AS-regularity is preserved by twisting, $\mathcal{D}\left(w^{\theta}\right)$ is AS-regular; that is, the MS twist $w^{\theta}$ is regular.

EXAMPLE 2.17. We set $w:=(x y z+y z x+z x y)-(z y x+y x z+x z y) \in V^{\otimes 3}$. Then we see that $\varphi(w)=w$. So, $w$ is a superpotential. The derivation-quotient algebra of $w$ is

$$
\begin{aligned}
\mathcal{D}(w) & =k\langle x, y, z\rangle /\left(\partial_{x} w, \partial_{y} w, \partial_{z} w\right) \\
& =k\langle x, y, z\rangle /(y z-z y, z x-x z, x y-y x)=k[x, y, z] .
\end{aligned}
$$


It is known that a polynomial ring $k[x, y, z]$ is Calabi-Yau AS-regular. So, by Definition $2.13(2), w$ is a Calabi-Yau superpotential. We take

$$
\theta:=\left(\begin{array}{ccc}
\alpha & 0 & 0 \\
0 & \beta & 0 \\
0 & 0 & \gamma
\end{array}\right)
$$

in $\mathrm{GL}_{3}(k)$. Calculating the MS twist $w^{\theta}$ of the superpotential $w$ by $\theta$, we obtain

$$
\begin{aligned}
w^{\theta} & =\left(\theta^{2} \otimes \theta \otimes \mathrm{id}\right)(w) \\
& =\left(\alpha^{2} \beta x y z+\beta^{2} \gamma y z x+\alpha \gamma^{2} z x y\right)-\left(\beta \gamma^{2} z y x+\alpha \beta^{2} y x z+\alpha^{2} \gamma x z y\right) .
\end{aligned}
$$

Therefore, the derivation-quotient algebra of $w^{\theta}$ is

$$
\begin{aligned}
\mathcal{D}\left(w^{\theta}\right) & =k\langle x, y, z\rangle /\left(\partial_{x} w, \partial_{y} w, \partial_{z} w\right) \\
& =k\langle x, y, z\rangle /\left(\alpha^{2} \beta y z-\alpha^{2} \gamma z y, \beta^{2} \gamma z x-\alpha \beta^{2} x z, \alpha \gamma^{2} x y-\beta \gamma^{2} y x\right) \\
& =k\langle x, y, z\rangle /(\beta y z-\gamma z y, \gamma z x-\alpha x z, \alpha x y-\beta y x) .
\end{aligned}
$$

Since Aut $(w)=\mathrm{GL}(V)$, we see that $\theta \in \operatorname{Aut}(w)$. By Lemma 2.16, $w^{\theta}$ is a regular twisted superpotential, so, $\mathcal{D}\left(w^{\theta}\right)$ is an AS-regular algebra.

2.4. Geometric algebras. Let $\left(E, O_{E}\right)$ be a scheme where $O_{E}$ is the structure sheaf on $E$. An invertible sheaf on $E$ is defined to be a locally free $O_{E}$-module of rank 1 . For a quadratic algebra $A=T(V) /(R)$, we set

$$
\mathcal{V}(R):=\left\{(p, q) \in \mathbb{P}\left(V^{*}\right) \times \mathbb{P}\left(V^{*}\right) \mid f(p, q)=0 \text { for all } f \in R\right\} .
$$

Let $E \subset \mathbb{P}\left(V^{*}\right)$ be a closed $k$-subscheme and $\sigma$ an automorphism of $E$. For the rest of the paper, we fix

(a) $\pi: E \rightarrow \mathbb{P}\left(V^{*}\right)$ is the embedding,

(b) $\mathcal{L}:=\pi^{*}\left(O_{\mathbb{P}\left(V^{*}\right)}(1)\right)$.

In this case, $\mathcal{L}$ becomes an invertible sheaf on $E$. The map

$$
\mu: \mathrm{H}^{0}(E, \mathcal{L}) \otimes \mathrm{H}^{0}(E, \mathcal{L}) \rightarrow \mathrm{H}^{0}(E, \mathcal{L}) \otimes \mathrm{H}^{0}\left(E, \mathcal{L}^{\sigma}\right) \rightarrow \mathrm{H}^{0}\left(E, \mathcal{L} \otimes_{O_{E}} \mathcal{L}^{\sigma}\right)
$$

of $k$-vector spaces is defined by $v \otimes w \mapsto v \otimes w^{\sigma}$ where $\mathcal{L}^{\sigma}=\sigma^{*} \mathcal{L}$ and $w^{\sigma}=w \circ \sigma$.

For a quadratic algebra, a geometric algebra was introduced by Mori [12].

Definition 2.18 [12, Definition 4.3]. A quadratic algebra $A=T(V) /(R)$ is called geometric if there is a pair $(E, \sigma)$ where $E \subset \mathbb{P}\left(V^{*}\right)$ is a closed $k$-subscheme, and $\sigma$ is a $k$-automorphism of $E$ such that

- $(\mathrm{G} 1): \mathcal{V}(R)=\left\{(p, \sigma(p)) \in \mathbb{P}\left(V^{*}\right) \times \mathbb{P}\left(V^{*}\right) \mid p \in E\right\}$, and

- (G2): $R=\operatorname{ker} \mu$ with the identification

$$
\mathrm{H}^{0}(E, \mathcal{L})=\mathrm{H}^{0}\left(\mathbb{P}\left(V^{*}\right), \mathcal{O}_{\mathbb{P}\left(V^{*}\right)}(1)\right)=V \quad \text { as } k \text {-vector spaces. }
$$

When $A$ satisfies condition (G2), we write $A=\mathcal{A}(E, \sigma)$. 
Let $A=T(V) /(R)$ be a quadratic algebra. If $A=\mathcal{A}(E, \sigma)$ is a geometric algebra, then $E$ is called the point scheme of $A$. If $E$ is reduced, then condition (G2) is equivalent to condition $\left(\mathrm{G}^{\prime}\right)$ :

(see [12]).

$$
R=\left\{f \in V \otimes V|f|_{\mathcal{V}(R)}=0\right\}
$$

TheOREM 2.19 [3, Theorem 3]. Let $A$ be a quadratic algebra. Then $A$ is a three-dimensional AS-regular algebra if and only if $A$ is isomorphic to a geometric algebra $\mathcal{A}(E, \sigma)$ that satisfies one of the following conditions:

(1) $E=\mathbb{P}^{2}$ and $\sigma \in$ Aut $_{k} \mathbb{P}^{2}$.

(2) E is a cubic curve in $\mathbb{P}^{2}$ and $\sigma \in$ Aut $_{k}$ E such that $\sigma^{*} \mathcal{L} ¥ \mathcal{L}$ and

$$
\left(\sigma^{2}\right)^{*} \mathcal{L} \otimes_{O_{E}} \mathcal{L} \cong \sigma^{*} \mathcal{L} \otimes_{O_{E}} \sigma^{*} \mathcal{L} \text {. }
$$

The types of $(E, \sigma)$ of three-dimensional quadratic AS-regular algebras are defined in [15]; these are slightly modified from the original types defined in [1]. We extend the types defined in [15] as follows (see [10, Subsection 2.3]).

(1) Type P: $E$ is $\mathbb{P}^{2}$, and $\sigma \in \operatorname{Aut}_{k} \mathbb{P}^{2}=\operatorname{PGL}_{3}(k)$ (Type $\mathrm{P}$ is divided into Types $\mathrm{P}_{i}(i=$ $1,2,3$ ) in terms of the Jordan canonical form of $\sigma$ ).

(2-1) Type $\mathrm{S}_{1}: E$ is a triangle, and $\sigma$ stabilizes each component.

(2-2) Type $\mathrm{S}_{2}: E$ is a triangle, and $\sigma$ interchanges two of its components.

(2-3) Type $\mathrm{S}_{3}: E$ is a triangle, and $\sigma$ circulates three components.

(3-1) Type $S_{1}^{\prime}: E$ is a union of a line and a conic meeting at two points, and $\sigma$ stabilizes each component and two intersection points.

(3-2) Type $\mathrm{S}_{2}^{\prime}: E$ is a union of a line and a conic meeting at two points, and $\sigma$ stabilizes each component and interchanges two intersection points.

(4-1) Type $\mathrm{T}_{1}: E$ is a union of three lines meeting at one point, and $\sigma$ stabilizes each component.

(4-2) Type $\mathrm{T}_{2}: E$ is a union of three lines meeting at one point, and $\sigma$ interchanges two of its components.

(4-3) Type $\mathrm{T}_{3}: E$ is a union of three lines meeting at one point, and $\sigma$ circulates three components.

(5) Type $\mathrm{T}^{\prime}: E$ is a union of a line and a conic meeting at one point, and $\sigma$ stabilizes each component.

(6) Type CC: $E$ is a cuspidal cubic curve.

(7) Type NC: $E$ is a nodal cubic curve (Type NC is divided into Types $\mathrm{NC}_{i}(i=1,2)$ ).

(8) Type WL: $E$ is a union of a double line and a line (Type WL is divided into Types $\left.\mathrm{WL}_{i}(i=1,2,3)\right)$. 
(9) Type TL: $E$ is a triple line (Type TL is divided into Types $\mathrm{TL}_{i}(i=1,2,3,4)$ ).

(10) Type EC: $E$ is an elliptic curve.

REMARK 2.20. All possible defining relations of three-dimensional quadratic AS-regular algebras are listed in each type up to isomorphism from (1) to (9) in [10, Theorem 3.1], and (10) in [10, Theorem 4.9].

\section{Classifications of twisted superpotentials}

In this section we give complete lists of superpotentials and twisted superpotentials whose derivation-quotient algebras are three-dimensional quadratic AS-regular algebras except for Type EC, by using the following three steps.

Step I. (Proposition 3.1) Find the candidates of regular twisted superpotentials corresponding to defining relations listed in [10, Theorem 3.1].

Step II. (Theorem 3.3) Find all superpotentials among the above candidates and show that they are Calabi-Yau superpotentials.

Step III. (Theorems 3.4 and 3.5) Show that all above candidates can be written as MS twists of Calabi-Yau superpotentials and that they are in fact regular twisted superpotentials.

As a byproduct, we prove that, for any three-dimensional quadratic AS-regular algebra $A$ except for Type EC, there exist a Calabi-Yau AS-regular algebra $S$ and $\theta \in$ Aut $S$ such that $A$ is isomorphic to $S^{\theta}$ as graded $k$-algebras. This result is needed to prove our main result, Theorem 1.1 .

PROPOSITION 3.1. Every three-dimensional quadratic AS-regular algebra except for Type EC is isomorphic to $\mathcal{D}(w)$ of a potential $w$ in Table 1.

Proof. In [10, Theorem 3.1], all possible defining relations $f_{1}, f_{2}, f_{3}$ of threedimensional quadratic AS-regular algebras except for Type EC are given. In each type, it is enough to find $w$ such that $\left(\partial_{x} w, \partial_{y} w, \partial_{z} w\right)=\left(f_{1}, f_{2}, f_{3}\right)$.

TABLE 1. List of potential $w$ and the Nakayama automorphism of the quadratic dual.

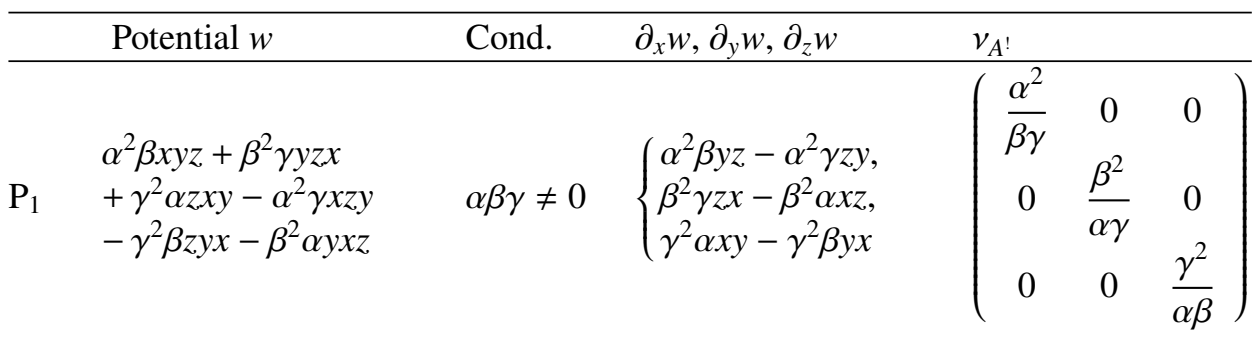


TABle 1. Continued.

\begin{tabular}{|c|c|c|c|c|}
\hline $\mathrm{P}_{2}$ & $\begin{array}{l}x y z+\alpha y z x+\alpha^{2} z x y \\
-\alpha x z y-\alpha^{2} z y x \\
-y x z+y^{2} z-2 \alpha y z y \\
+\alpha^{2} z y^{2}\end{array}$ & $\alpha \neq 0$ & $\left\{\begin{array}{r}y z-\alpha z y \\
y z-2 \alpha z y+\alpha z x \\
-x z \\
\alpha^{2} y^{2}+\alpha^{2} x y \\
-\alpha^{2} y x\end{array}\right.$ & $\left(\begin{array}{ccc}\frac{1}{\alpha} & \frac{3}{\alpha} & 0 \\
0 & \frac{1}{\alpha} & 0 \\
0 & 0 & \alpha^{2}\end{array}\right)$ \\
\hline $\mathrm{P}_{3}$ & $\begin{array}{l}-x y z-y z x-z x y \\
+x z y+z y x+y x z \\
-z^{2} x+2 z x z-x z^{2} \\
-z y^{2}+z y z+z^{2} y \\
-y^{2} z+2 y z y-2 y z^{2}-z^{3}\end{array}$ & 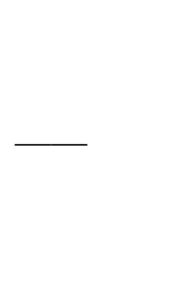 & $\left\{\begin{array}{c}z y-y z-z^{2} \\
x z-y z-2 z^{2} \\
-z x+2 z y \\
-x y+y x-y^{2} \\
-z x+2 x z+y z \\
+z y-z^{2}\end{array}\right.$ & $\left(\begin{array}{lll}1 & 3 & 3 \\
0 & 1 & 3 \\
0 & 0 & 1\end{array}\right)$ \\
\hline $\mathrm{S}_{1}$ & $\begin{array}{l}\beta x y z+\gamma y z x+\alpha z x y \\
-\alpha \beta x z y-\alpha \gamma z y x \\
-\beta \gamma y x z\end{array}$ & $\alpha \beta \gamma \neq 0,1$ & $\left\{\begin{array}{l}\beta y z-\alpha \beta z y \\
\gamma z x-\beta \gamma x z \\
\alpha x y-\alpha \gamma y x\end{array}\right.$ & $\left(\begin{array}{ccc}\frac{\beta}{\gamma} & 0 & 0 \\
0 & \frac{\gamma}{\alpha} & 0 \\
0 & 0 & \frac{\alpha}{\beta}\end{array}\right)$ \\
\hline $\mathrm{S}_{2}$ & $\begin{array}{l}-y z x-x z y+\frac{1}{\beta} x^{2} z \\
+\frac{1}{\alpha} z x^{2}+\alpha y^{2} z \\
+\beta z y^{2}\end{array}$ & $\alpha \beta \neq 0$ & $\left\{\begin{array}{l}\frac{1}{\beta} x z-z y \\
\alpha y z-z x \\
\frac{1}{\alpha} x^{2}+\beta y^{2}\end{array}\right.$ & $\left(\begin{array}{ccc}0 & -\alpha & 0 \\
-\frac{1}{\beta} & 0 & 0 \\
0 & 0 & \frac{\beta}{\alpha}\end{array}\right)$ \\
\hline $\mathrm{S}_{3}$ & $\begin{array}{l}-x z y-z y x \\
-y x z+\beta x^{3} \\
+\gamma y^{3}+\alpha z^{3}\end{array}$ & $\alpha \beta \gamma \neq 0,1$ & $\left\{\begin{array}{l}\beta x^{2}-z y \\
\gamma y^{2}-x z \\
\alpha z^{2}-y x\end{array}\right.$ & $\left(\begin{array}{lll}1 & 0 & 0 \\
0 & 1 & 0 \\
0 & 0 & 1\end{array}\right)$ \\
\hline$S_{1}^{\prime}$ & $\begin{array}{l}\beta x y z+\beta y z x \\
+\alpha z x y-\alpha \beta x z y \\
-\alpha \beta z y x-\beta^{2} y x z \\
+\beta x^{3}\end{array}$ & $\alpha \beta^{2} \neq 0,1$ & $\left\{\begin{array}{l}\beta x^{2}+\beta y z \\
-\alpha \beta z y \\
\beta z x-\beta^{2} x z \\
\alpha x y-\alpha \beta y x\end{array}\right.$ & $\left(\begin{array}{ccc}1 & 0 & 0 \\
0 & \frac{\beta}{\alpha} & 0 \\
0 & 0 & \frac{\alpha}{\beta}\end{array}\right)$ \\
\hline $\mathrm{S}_{2}^{\prime}$ & $\begin{array}{l}-z x y-y x z \\
+x y^{2}+y^{2} x \\
+x z^{2}+z^{2} x+x^{3}\end{array}$ & - & $\left\{\begin{array}{l}x^{2}+y^{2}+z^{2} \\
y x-x z \\
z x-x y\end{array}\right.$ & $\left(\begin{array}{ccc}1 & 0 & 0 \\
0 & 0 & -1 \\
0 & -1 & 0\end{array}\right)$ \\
\hline $\mathrm{T}_{1}$ & $\begin{array}{l}\beta x^{2} y \\
+(\alpha-\beta+\gamma) x y x \\
+(\alpha-\beta-\gamma) y x y \\
-\alpha y^{2} x-y x z \\
+y z x+\beta y x^{2} \\
+x y z-x z y \\
-\alpha x y^{2}+z x y-z y x\end{array}$ & $\begin{array}{l}\alpha+\beta+ \\
\gamma \neq 0\end{array}$ & $\left\{\begin{array}{r}\beta x y+(\alpha-\beta+\gamma) y x \\
+y z-z y-\alpha y^{2}, \\
(\alpha-\beta-\gamma) x y-\alpha y x \\
-x z+z x+\beta x^{2}, \\
x y-y x\end{array}\right.$ & $\begin{array}{l}\left(\begin{array}{lll}1 & 0 & 0 \\
0 & 1 & 0 \\
\delta & \varepsilon & 1\end{array}\right) \\
\delta:=-\alpha+2 \beta-\gamma, \\
\varepsilon:=2 \alpha-\beta-\gamma\end{array}$ \\
\hline
\end{tabular}


TABle 1. Continued.

\begin{tabular}{|c|c|c|c|c|}
\hline $\mathrm{T}_{2}$ & $\begin{array}{l}(1-\beta-\gamma) x^{3} \\
-(\alpha+2 \gamma) y x^{2} \\
+z x^{2}-x y^{2} \\
+\gamma y^{3}-z y^{2} \\
-x^{2} z+x z y \\
+\beta x^{2} y-y^{2} z \\
+y z x+\alpha y^{2} x\end{array}$ & $\begin{array}{l}\alpha+\beta+ \\
\gamma \neq 0\end{array}$ & $\left\{\begin{array}{r}(1-\beta-\gamma) x^{2}-y^{2} \\
-x z+z y+\beta x y \\
-(\alpha+2 \gamma) x^{2}+\gamma y^{2} \\
-y z+z x+\alpha y x \\
x^{2}-y^{2}\end{array}\right.$ & 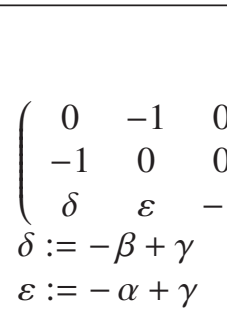 \\
\hline $\mathrm{T}_{3}$ & $\begin{array}{l}-x^{3}+y^{3}+x^{2} y \\
+x y x+y x^{2} \\
-x y^{2}-y x y \\
-y^{2} x+x^{2} z \\
+x z x+z x^{2} \\
+z y^{2}+y z y \\
+y^{2} z-x y z \\
-y z x-z x y\end{array}$ & - & $\left\{\begin{array}{c}-x^{2}+x y+y x-y^{2} \\
+x z+z x-y z \\
y^{2}+x^{2}-x y-y x \\
\quad+z y+y z-z x \\
x^{2}+y^{2}-x y\end{array}\right.$ & $\left(\begin{array}{lll}1 & 0 & 0 \\
0 & 1 & 0 \\
0 & 0 & 1\end{array}\right)$ \\
\hline $\mathrm{T}^{\prime}$ & $\begin{array}{l}\alpha x^{2} y- \\
(\alpha-2 \beta) x y x \\
+\left(\beta^{2}-\alpha \beta\right) x y^{2} \\
+x y z-x z y \\
+\alpha y x^{2}-y x z \\
+y z x-\alpha y z y \\
+\alpha \beta^{2} y^{3} \\
-\left(\beta^{2}-\alpha \beta\right) y^{2} x \\
-\beta y^{2} z+z x y \\
-z y x-\beta z y^{2}\end{array}$ & $\alpha+2 \beta \neq 0$ & $\left\{\begin{array}{c}\alpha x y-(\alpha-2 \beta) y x \\
+\left(\beta^{2}-\alpha \beta\right) y^{2} \\
+y z-z y, \\
\alpha x^{2}-x z+z x \\
-\alpha z y+\alpha \beta^{2} y^{2} \\
-\left(\beta^{2}-\alpha \beta\right) y x \\
-\beta y z \\
x y-y x-\beta y^{2}\end{array}\right.$ & $\begin{array}{l}\left(\begin{array}{ccc}1 & \delta & 0 \\
0 & 1 & 0 \\
2 \delta & \delta^{2} & 1\end{array}\right) \\
\delta:=\alpha-\beta\end{array}$ \\
\hline $\mathrm{CC}$ & $\begin{array}{l}-3 x^{3}-y^{2} x \\
-y x y-x y^{2} \\
+y^{2} z+y z y \\
+z y^{2}-x y z \\
-y z x-z x y \\
+x z y+z y x \\
+y x z\end{array}$ & - & $\left\{\begin{array}{l}-3 x^{2}-y^{2} \\
-y z+z y \\
-y x-x y+y z \\
+z y-z x+x z \\
y^{2}-x y+y x\end{array}\right.$ & $\left(\begin{array}{lll}1 & 0 & 0 \\
0 & 1 & 0 \\
0 & 0 & 1\end{array}\right)$ \\
\hline $\mathrm{NC}_{1}$ & $\begin{array}{l}\frac{1-\alpha^{3}}{\alpha} x^{3} \\
+\frac{1-\alpha^{3}}{\alpha} y^{3} \\
+x y z+y z x \\
+z x y \\
-\alpha(x z y+z y x+y x z)\end{array}$ & $\alpha^{3} \neq 0,1$ & $\left\{\begin{array}{l}\frac{1-\alpha^{3}}{\alpha} x^{2}+y z-\alpha z y, \\
\frac{1-\alpha^{3}}{\alpha} y^{2}+z x-\alpha x z, \\
x y-\alpha y x\end{array}\right.$ & $\left.\begin{array}{lll}1 & 0 & 0 \\
0 & 1 & 0 \\
0 & 0 & 1\end{array}\right)$ \\
\hline
\end{tabular}


TABLE 1. Continued.

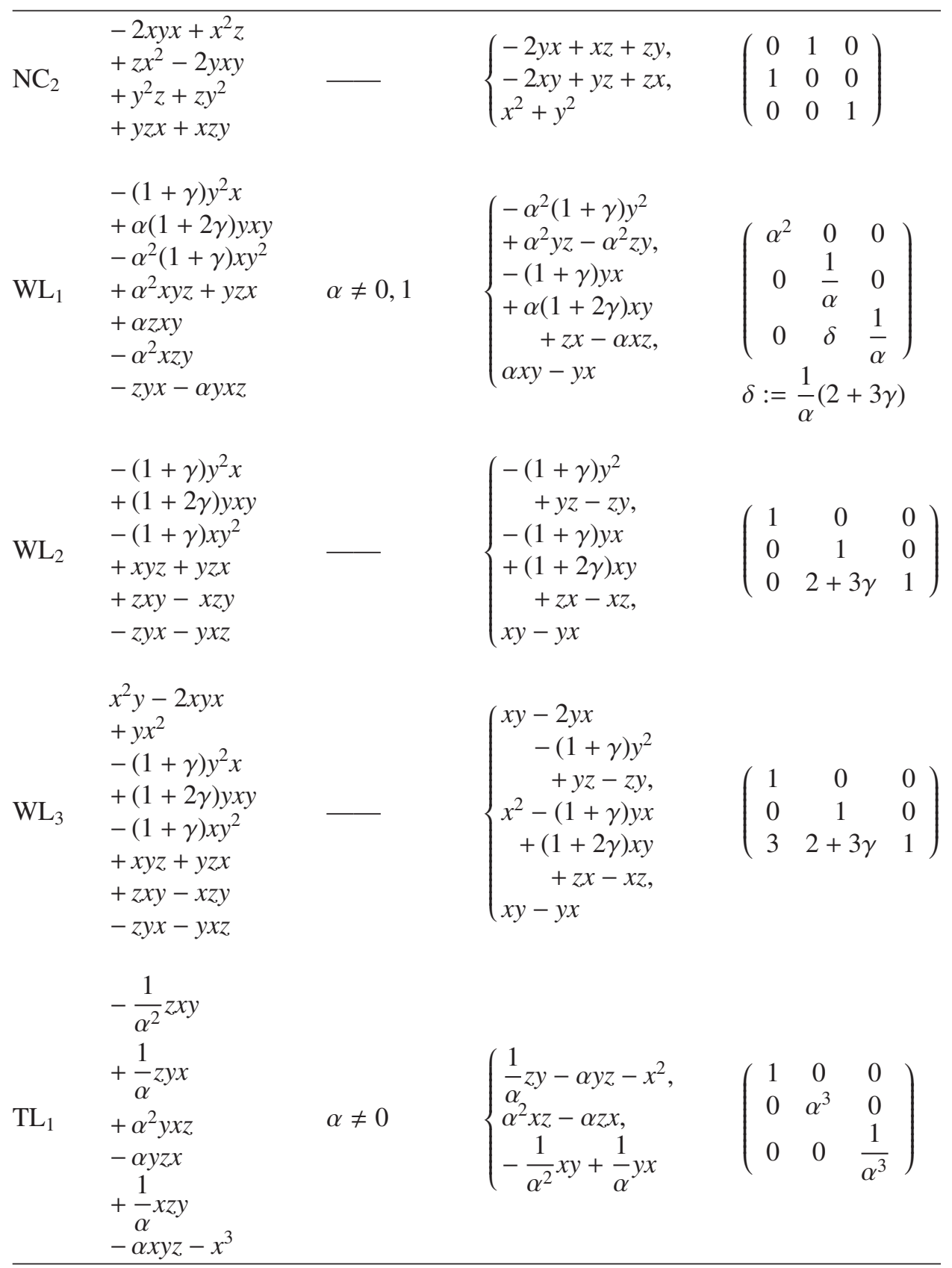


TABle 1. Continued.

\begin{tabular}{|c|c|c|c|c|c|}
\hline $\mathrm{TL}_{2}$ & $\begin{array}{l}\beta x^{2} y+\beta x y x \\
+\left(-\beta^{2}-1\right) x^{3} \\
+2 y x y-y^{2} x \\
-2 \beta y x^{2}+z x y \\
-z y x-\beta z x^{2} \\
-\beta x^{2} z+2 \beta x z x \\
-y x z+y z x \\
-x z y+x y z-x y^{2}\end{array}$ & $\left\{\begin{array}{c}\beta x y+\beta y x \\
+\left(-\beta^{2}-1\right) x^{2} \\
-\beta x z+2 \beta z x \\
-z y+y z-y^{2}, \\
2 x y-y x-2 \beta x^{2} \\
-x z+z x, \\
x y-y x-\beta x^{2}\end{array}\right.$ & $\begin{array}{l}1 \\
3 \\
3\end{array}$ & 3 & $\left.\begin{array}{l}0 \\
0 \\
1\end{array}\right)$ \\
\hline $\mathrm{TL}_{3}$ & $\begin{array}{l}-2 y x y-y^{2} x \\
+z x y+z y x \\
-y x z-y z x \\
+x z y-x y z \\
-x^{3}-x y^{2}\end{array}$ & $\left\{\begin{array}{l}z y-y z \\
-x^{2}-y^{2} \\
-2 x y-y x \\
-x z-z x \\
x y+y x\end{array}\right.$ & $\begin{array}{l}1 \\
0 \\
0\end{array}$ & $\begin{array}{r}0 \\
- \\
3\end{array}$ & $\begin{array}{c}0 \\
0 \\
-1\end{array}$ \\
\hline $\mathrm{TL}_{4}$ & $\begin{array}{l}-x^{3}+y x^{2} \\
+x^{2} y-2 x y x \\
+z x y+x y z \\
+y z x-z y x \\
-y x z-x z y\end{array}$ & $\left\{\begin{array}{l}-x^{2}+x y-2 y x \\
\quad+y z-z y, \\
x^{2}+z x-x z \\
x y-y x\end{array}\right.$ & $\begin{array}{l}1 \\
0 \\
3\end{array}$ & $\begin{array}{l}0 \\
1 \\
0\end{array}$ & $\left.\begin{array}{l}0 \\
0 \\
1\end{array}\right)$ \\
\hline
\end{tabular}

We give a proof for Type $\mathrm{T}_{2}$ algebras. For the other types, the proofs are similar. From [10, Theorem 3.1], Type $\mathrm{T}_{2}$ algebras are given as $A=k\langle x, y, z\rangle /\left(f_{1}, f_{2}, f_{3}\right)$ :

$$
\left\{\begin{array}{l}
f_{1}=x^{2}-y^{2} \\
f_{2}=x z-z y-\beta x y+(\beta+\gamma) y^{2}, \\
f_{3}=y z-z x-\alpha y x+(\alpha+\gamma) x^{2}
\end{array}\right.
$$

where $\alpha+\beta+\gamma \neq 0$. Taking a potential

$$
\begin{aligned}
w= & (1-\beta-\gamma) x^{3}-(\alpha+2 \gamma) y x^{2}+z x^{2}-x y^{2}+\gamma y^{3}-z y^{2} \\
& -x^{2} z+x z y+\beta x^{2} y-y^{2} z+y z x+\alpha y^{2} x
\end{aligned}
$$

as in Table 1, we have

$$
\left\{\begin{array}{l}
\partial_{x} w=(1-\beta-\gamma) x^{2}-y^{2}-x z+z y+\beta x y \\
\partial_{y} w=-(\alpha+2 \gamma) x^{2}+\gamma y^{2}-y z+z x+\alpha y x \\
\partial_{z} w=x^{2}-y^{2}
\end{array}\right.
$$

Since $\partial_{z} w=x^{2}-y^{2}=f_{1}, \partial_{x} w=(1-\beta-\gamma) f_{1}-f_{2}$ and $\partial_{y} w=-\gamma f_{1}-f_{3}$, it follows that $\mathcal{D}(w)=k\langle x, y, z\rangle /\left(\partial_{x} w, \partial_{y} w, \partial_{z} w\right)=k\langle x, y, z\rangle /\left(f_{1}, f_{2}, f_{3}\right)=A$.

REMARK 3.2. (1) For an algebra $A=k\langle x, y, z\rangle /\left(f_{1}, f_{2}, f_{3}\right)$ of any type, we can take a potential $w=x f_{1}+y f_{2}+z f_{3}$ such that $A=\mathcal{D}(w)$. But it is difficult to check that this 
potential $w$ is a regular twisted superpotential and, in many cases, it is not so. The potentials $w$ listed in Proposition 3.1 are chosen so that they are candidates of regular twisted superpotentials. By [13, Theorem 4.4], every regular twisted superpotential $w$ satisfies $\left(v^{-1} \otimes \mathrm{id} \otimes \mathrm{id}\right)(\varphi(w))=w$ where $v$ is the Nakayama automorphism of $A^{!}$, so in the above proposition, we take a potential $w$ such that

$$
\left(\left(v_{A} !\right)^{-1} \otimes \mathrm{id} \otimes \mathrm{id}\right)(\varphi(w))=w
$$

where $v_{A}$ is the Nakayama automorphism of $A^{!}$listed in Table 1.

(2) For Type $\mathrm{TL}_{4}$ in [10, Theorem 3.1], we gave the defining relations

$$
\left\{\begin{array}{l}
x y+y x \\
x z-z x-x^{2} \\
z y-y z+x y+x^{2}
\end{array}\right.
$$

The relation $x y+y x$ is a typo, and the relation $x y-y x$ is correct, as given in Table 1 .

Next, we give a complete list of Calabi-Yau superpotentials as follows.

THEOREM 3.3. For every type except for Type EC, Table 2 is a complete list of Calabi-Yau superpotentials $w_{0}$.

Proof. Let $A$ be a three-dimensional quadratic AS-regular algebra except for Type EC. By Lemma 2.4, $A$ is Calabi-Yau if and only if the Nakayama automorphism $v_{A}$ ! is the identity, where $A^{!}$is the quadratic dual of $A$. Considering the condition that $v_{A}$ ! in Table 1 is the identity, we have a superpotential, so it is sufficient to show that $w_{0}$ is regular; that is, $\mathcal{D}\left(w_{0}\right)=k\langle x, y, z\rangle /\left(\partial_{x} w_{0}, \partial_{y} w_{0}, \partial_{z} w_{0}\right)$ is a three-dimensional quadratic AS-regular algebra. In fact, if $w$ is regular, then by Lemma 2.15, it is Calabi-Yau. In order to prove AS-regularity of $\mathcal{D}\left(w_{0}\right)$, we check that $\mathcal{D}\left(w_{0}\right)$ satisfies the conditions of Theorem 2.2. Note that if $w$ is a superpotential, then the derivation-quotient algebra $\mathcal{D}(w)$ is standard if and only if the partial derivatives $\partial_{x} w, \partial_{y} w, \partial_{z} w$ are linearly independent (see, for example, [14, Proposition 2.6]).

We give a proof for Type $\mathrm{T}_{1}$ algebras. For the other types, the proofs are similar. Let

$$
w_{0}=x y z+y z x+z x y-(x z y+z y x+y x z)+\left(x^{2} y+x y x+y x^{2}\right)-\left(y^{2} x+y x y+x y^{2}\right) .
$$

It is easy to check that $\partial_{x} w_{0}, \partial_{y} w_{0}, \partial_{z} w_{0}$ are linearly independent. For the potential $w_{0}$, we have the unique $3 \times 3$ matrix

$$
M:=\left(\begin{array}{ccc}
y & x-y-z & y \\
x-y+z & -x & -x \\
-y & x & 0
\end{array}\right) \quad \text { such that }\left(\begin{array}{c}
\partial_{x} w_{0} \\
\partial_{y} w_{0} \\
\partial_{z} w_{0}
\end{array}\right)=M\left(\begin{array}{l}
x \\
y \\
z
\end{array}\right)
$$

By calculation, we have $\left(\begin{array}{lll}x & y & z\end{array}\right) M=\left(\begin{array}{lll}\partial_{x} w_{0} & \partial_{y} w_{0} & \partial_{z} w_{0}\end{array}\right)$. Hence, $\mathcal{D}\left(w_{0}\right)$ is standard. We denote by $\Delta_{i j}$ the $(i, j)$ th $2 \times 2$ minors of the matrix $M(1 \leq i, j \leq 3)$. Since $\Delta_{11}=$ $-x^{2}, \Delta_{22}=y^{2}$ and $\Delta_{33}=-x^{2}+x y-y^{2}+z^{2}$, we have that $\mathcal{V}\left(\left\{\Delta_{i j} \mid 1 \leq i, j \leq 3\right\}\right)=\emptyset$. 
Therefore, by Theorem 2.2, $\mathcal{D}\left(w_{0}\right)$ is a three-dimensional quadratic AS-regular algebra, that is, $w_{0}$ is a Calabi-Yau superpotential.

THEOREM 3.4. For a potential $w$ in Table 1, there exist a Calabi-Yau superpotential $w_{0}$ in Table 2 and $\theta \in$ Aut $\left(w_{0}\right)$ such that $\mathcal{D}(w) \cong \mathcal{D}\left(\left(w_{0}\right)^{\theta}\right)$ as in Table 3 .

Proof. By direct computation, for a potential $w$ in Table 1, we find a Calabi-Yau superpotential $w_{0}$ in Table 2 and $\theta \in \operatorname{Aut}\left(w_{0}\right)$ such that $\mathcal{D}(w) \cong \mathcal{D}\left(\left(w_{0}\right)^{\theta}\right)$ as in Table 3.

We give a proof for Type $T_{1}$ algebra. For the other types, the proofs are similar. Let $w$ be a potential of Type $\mathrm{T}_{1}$ in Table 1 . We take a superpotential

$$
\begin{aligned}
w_{0}= & x y z+y z x+z x y-(x z y+y x z+z y x) \\
& +\left(x^{2} y+x y x+y x^{2}\right)-\left(x y^{2}+y x y+y^{2} x\right)
\end{aligned}
$$

TABLE 2. List of Calabi-Yau superpotentials and defining relations of the derivation-quotient

\begin{tabular}{|c|c|c|c|}
\hline & CY superpotential $w_{0}$ & Cond. & $\partial_{x} w_{0}, \partial_{y} w_{0}, \partial_{z} w_{0}$ \\
\hline$P_{1}$ & $\begin{array}{l}x y z+y z x+z x y \\
-\alpha(x z y+z y x+y x z)\end{array}$ & $\alpha^{3}=1$ & $\left\{\begin{array}{l}y z-\alpha z y \\
z x-\alpha x z \\
x y-\alpha y x\end{array}\right.$ \\
\hline $\mathrm{S}_{1}$ & $\begin{array}{l}x y z+y z x+z x y \\
-\alpha(x z y+z y x+y x z)\end{array}$ & $\alpha^{3} \neq 0,1$ & $\left\{\begin{array}{l}y z-\alpha z y \\
z x-\alpha x z \\
x y-\alpha y x\end{array}\right.$ \\
\hline $\mathrm{S}_{3}$ & $x z y+z y x+y x z-\alpha\left(x^{3}+y^{3}+z^{3}\right)$ & $\alpha^{3} \neq 0,1$ & $\left\{\begin{array}{l}z y-\alpha x^{2} \\
x z-\alpha y^{2} \\
y x-\alpha z^{2}\end{array}\right.$ \\
\hline$S_{1}^{\prime}$ & $\begin{array}{l}x y z+y z x+z x y \\
-\alpha(x z y+z y x+y x z)+x^{3}\end{array}$ & $\alpha^{3} \neq 0,1$ & $\left\{\begin{array}{l}y z-\alpha z y+x^{2} \\
z x-\alpha x z \\
x y-\alpha y x\end{array}\right.$ \\
\hline $\mathrm{T}_{1}$ & $\begin{array}{l}x y z+y z x+z x y \\
-(x z y+z y x+y x z) \\
+\left(x^{2} y+x y x+y x^{2}\right) \\
-\left(y^{2} x+y x y+x y^{2}\right)\end{array}$ & - & $\left\{\begin{array}{l}y z-z y+x y+y x-y^{2} \\
z x-x z+x^{2}-y x-x y, \\
x y-y x\end{array}\right.$ \\
\hline $\mathrm{T}_{3}$ & $\begin{array}{l}x y z+y z x+z x y \\
-\left(x^{2} y+x y x+y x^{2}\right) \\
+\left(x y^{2}+y x y+y^{2} x\right) \\
-\left(x^{2} z+x z x+z x^{2}\right) \\
-\left(z y^{2}+y z y+y^{2} z\right) \\
+x^{3}-y^{3}\end{array}$ & - & $\left\{\begin{array}{r}y z-x y-y x+y^{2}-x z \\
-z x+x^{2} \\
z x-x^{2}+x y+y x-z y \\
-y z-y^{2} \\
x y-x^{2}-y^{2}\end{array}\right.$ \\
\hline
\end{tabular}
algebra of $w_{0}$. 
TABle 2. Continued.

\begin{tabular}{|c|c|c|c|}
\hline $\mathrm{T}^{\prime}$ & $\begin{array}{l}x y z+y z x+z x y \\
-(x z y+z y x+y x z) \\
+\left(x^{2} y+x y x+y x^{2}\right) \\
-\left(y^{2} z+y z y+z y^{2}\right)+y^{3}\end{array}$ & & $\left\{\begin{array}{r}y z-z y+x y+y x \\
z x-x z+x^{2}-y z \\
-z y+y^{2} \\
x y-y x-y^{2}\end{array}\right.$ \\
\hline $\mathrm{CC}$ & $\begin{array}{l}x y z+y z x+z x y \\
-(x z y+z y x+y x z) \\
+\left(y^{2} x+y x y+x y^{2}\right) \\
-\left(y^{2} z+y z y+z y^{2}\right)+3 x^{3}\end{array}$ & & $\left\{\begin{array}{c}y z-z y+y^{2}+3 x^{2} \\
z x-x z+y x+x y \\
-y z-z y \\
x y-y x-y^{2}\end{array}\right.$ \\
\hline $\mathrm{NC}_{1}$ & $\begin{array}{l}x y z+y z x+z x y \\
-\alpha(x z y+z y x+y x z) \\
+x^{3}+y^{3}\end{array}$ & $\alpha^{3} \neq 0,1$ & $\left\{\begin{array}{l}y z-\alpha z y+x^{2} \\
z x-\alpha x z+y^{2} \\
x y-\alpha y x\end{array}\right.$ \\
\hline $\mathrm{WL}_{2}$ & $\begin{array}{l}x y z+y z x+z x y \\
-(x z y+z y x+y x z) \\
-\frac{1}{3}\left(y^{2} x+y x y+x y^{2}\right)\end{array}$ & $\longrightarrow$ & $\left\{\begin{array}{l}y z-z y-\frac{1}{3} y^{2} \\
z x-x z-\frac{1}{3}(y x+x y), \\
x y-y x\end{array}\right.$ \\
\hline $\mathrm{TL}_{1}$ & $\begin{array}{l}x y z+y z x+z x y \\
-\alpha(x z y+z y x+y x z)-x^{3}\end{array}$ & $\alpha^{3}=1$ & $\left\{\begin{array}{l}y z-\alpha z y-x^{2} \\
z x-\alpha x z \\
x y-\alpha y x\end{array}\right.$ \\
\hline
\end{tabular}

and

$$
\theta=\left(\begin{array}{ccc}
1 & 0 & 0 \\
0 & 1 & 0 \\
\lambda v^{-1} & \mu v^{-1} & 1
\end{array}\right)
$$

where $\lambda:=\frac{1}{3}(-\alpha+2 \beta-\gamma), \quad \mu:=\frac{1}{3}(2 \alpha-\beta-\gamma) \quad$ and $\quad v:=\frac{1}{3}(\alpha+\beta+\gamma)$. Since $\left(\theta^{\otimes 3}\right)\left(w_{0}\right)=w_{0}, \theta$ is in Aut $\left(w_{0}\right)$. By calculation, we have that

$$
\begin{aligned}
\left(w_{0}\right)^{\theta}= & (x y z+y z x+z x y)-(x z y+y x z+z y x) \\
& +v^{-1}\left(\beta x^{2} y+(\alpha-\beta+\gamma) x y x+\beta y x^{2}\right) \\
& -v^{-1}\left(\alpha y^{2} x+(-\alpha+\beta+\gamma) y x y+\alpha x y^{2}\right) .
\end{aligned}
$$

By taking

$$
\theta^{\prime}=\left(\begin{array}{ccc}
1 & 0 & 0 \\
0 & 1 & 0 \\
0 & 0 & v^{-1}
\end{array}\right) \in \mathrm{GL}_{3}(k)
$$

it follows that

$$
\left(\theta^{\otimes 3}\right)\left(\left(w_{0}\right)^{\theta}\right)=v^{-1} w
$$

so $\mathcal{D}\left(\left(w_{0}\right)^{\theta}\right) \cong \mathcal{D}(w)$. 
TABLE 3. List of Calabi-Yau superpotentials and automorphisms.

\begin{tabular}{|c|c|c|}
\hline & CY superpotential $w_{0}$ & $\theta \in \operatorname{Aut}\left(w_{0}\right)$ \\
\hline $\mathrm{P}_{1}$ & $\begin{array}{l}x y z+y z x+z x y-(x z y+z y x \\
+y x z)\end{array}$ & $\left(\begin{array}{lll}\alpha & 0 & 0 \\
0 & \beta & 0 \\
0 & 0 & \gamma\end{array}\right)$ \\
\hline $\mathrm{P}_{2}$ & $\begin{array}{l}x y z+y z x+z x y-(x z y+z y x \\
+y x z)\end{array}$ & $\left(\begin{array}{lll}1 & 1 & 0 \\
0 & 1 & 0 \\
0 & 0 & \alpha\end{array}\right)$ \\
\hline $\mathrm{P}_{3}$ & $\begin{array}{l}x y z+y z x+z x y-(x z y+z y x \\
+y x z)\end{array}$ & $\left(\begin{array}{lll}1 & 1 & 0 \\
0 & 1 & 1 \\
0 & 0 & 1\end{array}\right)$ \\
\hline $\mathrm{S}_{1}$ & $\begin{array}{l}(x y z+y z x+z x y)-\sqrt[3]{\alpha \beta \gamma}(x z y \\
+y x z+z y x)\end{array}$ & $\left(\begin{array}{ccc}\sqrt[3]{\beta \gamma^{-1}} & 0 & 0 \\
0 & \sqrt[3]{\gamma \alpha^{-1}} & 0 \\
0 & 0 & \sqrt[3]{\alpha \beta^{-1}}\end{array}\right)$ \\
\hline $\mathrm{S}_{2}$ & $\begin{array}{l}(x y z+y z x+z x y)+(x z y+y x z \\
+z y x)\end{array}$ & $\left(\begin{array}{ccc}0 & -\sqrt[3]{\alpha^{2} \beta} & 0 \\
-\frac{1}{\sqrt[3]{\alpha \beta^{2}}} & 0 & 0 \\
0 & 0 & \sqrt[3]{\beta \alpha^{-1}}\end{array}\right.$ \\
\hline $\mathrm{S}_{3}$ & $(x z y+y x z+z y x)-\alpha\left(x^{3}+y^{3}+z^{3}\right)$ & $\left(\begin{array}{lll}1 & 0 & 0 \\
0 & 1 & 0 \\
0 & 0 & 1\end{array}\right)$ \\
\hline$S_{1}^{\prime}$ & $\begin{array}{l}(x y z+y z x+z x y)-\sqrt[3]{\alpha \beta^{2}}(x z y \\
+y x z+z y x)+x^{3}\end{array}$ & $\left(\begin{array}{ccc}1 & 0 & 0 \\
0 & \sqrt[3]{\beta \alpha^{-1}} & 0 \\
0 & 0 & \sqrt[3]{\alpha \beta^{-1}}\end{array}\right.$ \\
\hline$S_{2}^{\prime}$ & $\begin{array}{l}(x y z+y z x+z x y)+(x z y+y x z \\
+z y x)+x^{3}\end{array}$ & $\left(\begin{array}{lll}1 & 0 & 0 \\
0 & 0 & 1 \\
0 & 1 & 0\end{array}\right)$ \\
\hline $\mathrm{T}_{1}$ & $\begin{array}{l}(x y z+y z x+z x y)-(x z y+y x z \\
+z y x)+\left(x^{2} y+x y x+y x^{2}\right)-\left(x y^{2}\right. \\
\left.+y x y+y^{2} x\right)\end{array}$ & $\begin{array}{l}\left(\begin{array}{ccc}1 & 0 & 0 \\
0 & 1 & 0 \\
\lambda v^{-1} & \mu v^{-1} & 1\end{array}\right) \\
\lambda:=\frac{1}{3}(-\alpha+2 \beta-\gamma), \\
\mu:=\frac{1}{3}(2 \alpha-\beta-\gamma), \\
\nu:=\frac{1}{3}(\alpha+\beta+\gamma)\end{array}$ \\
\hline
\end{tabular}


Table 3. Continued.

\begin{tabular}{|c|c|c|}
\hline $\mathrm{T}_{2}$ & $\begin{array}{l}(x y z+y z x+z x y)-(x z y+y x z \\
+z y x)+\left(x^{2} y+x y x+y x^{2}\right)-\left(x y^{2}\right. \\
\left.+y x y+y^{2} x\right)\end{array}$ & $\begin{array}{l}\left(\begin{array}{ccc}0 & -1 & 0 \\
-1 & 0 & 0 \\
-\lambda \nu^{-1} & -\mu v^{-1} & -1\end{array}\right) \\
\lambda:=\frac{1}{3}(-\alpha+2 \beta-\gamma), \\
\mu:=\frac{1}{3}(2 \alpha-\beta-\gamma), \\
\nu:=\frac{1}{3}(\alpha+\beta+\gamma)\end{array}$ \\
\hline $\mathrm{T}_{3}$ & $\begin{array}{l}(x y z+y z x+z x y)-\left(x^{2} y+x y x\right. \\
\left.+y x^{2}\right)+\left(x y^{2}+y x y+y^{2} x\right) \\
-\left(x^{2} z+x z x+z x^{2}\right)-\left(z y^{2}\right. \\
\left.+y z y+y^{2} z\right)+x^{3}-y^{3}\end{array}$ & $\left(\begin{array}{lll}1 & 0 & 0 \\
0 & 1 & 0 \\
0 & 0 & 1\end{array}\right)$ \\
\hline $\mathrm{T}^{\prime}$ & $\begin{array}{l}(x y z+y z x+z x y)-(x z y+y x z \\
+z y x)+\left(x^{2} y+x y x+y x^{2}\right)-\left(y^{2} z\right. \\
\left.+y z y+z y^{2}\right)+y^{3}\end{array}$ & $\begin{array}{l}\left(\begin{array}{ccc}1 & \lambda^{-1} \mu & 0 \\
0 & 1 & 0 \\
2 \lambda^{-1} \mu & \lambda^{-2} \mu^{2} & 1\end{array}\right) \\
\lambda:=\frac{1}{3}(\alpha+2 \beta), \mu:=\frac{1}{3}(\alpha-\beta)\end{array}$ \\
\hline $\mathrm{CC}$ & $\begin{array}{l}(x y z+y z x+z x y)-(x z y+y x z \\
+z y x)+\left(y^{2} x+y x y+x y^{2}\right) \\
-\left(y^{2} z+y z y+z y^{2}\right)+3 x^{3}\end{array}$ & $\left(\begin{array}{lll}1 & 0 & 0 \\
0 & 1 & 0 \\
0 & 0 & 1\end{array}\right)$ \\
\hline $\mathrm{NC}_{1}$ & $\begin{array}{l}(x y z+y z x+z x y)-\alpha(x z y+y x z \\
+z y x)+x^{3}+y^{3}\end{array}$ & $\left(\begin{array}{lll}1 & 0 & 0 \\
0 & 1 & 0 \\
0 & 0 & 1\end{array}\right)$ \\
\hline $\mathrm{NC}_{2}$ & $\begin{array}{l}(x y z+y z x+z x y)+(x z y+y x z \\
+z y x)+x^{3}+y^{3}\end{array}$ & $\left.\begin{array}{lll}0 & 1 & 0 \\
1 & 0 & 0 \\
0 & 0 & 1\end{array}\right)$ \\
\hline $\mathrm{WL}_{1}$ & $\begin{array}{l}x y z+y z x+z x y-(x z y+z y x \\
+y x z)-\frac{1}{3}\left(y^{2} x+y x y+x y^{2}\right)\end{array}$ & $\left(\begin{array}{ccc}\alpha & 0 & 0 \\
0 & 1 & 0 \\
0 & \frac{2}{3}+\gamma & 1\end{array}\right)$ \\
\hline $\mathrm{WL}_{2}$ & $\begin{array}{l}x y z+y z x+z x y-(x z y+z y x \\
+y x z)-\frac{1}{3}\left(y^{2} x+y x y+x y^{2}\right)\end{array}$ & $\left(\begin{array}{ccc}1 & 0 & 0 \\
0 & 1 & 0 \\
0 & \frac{2}{3}+\gamma & 1\end{array}\right)$ \\
\hline $\mathrm{WL}_{3}$ & $\begin{array}{l}x y z+y z x+z x y-(x z y+z y x \\
+y x z)-\frac{1}{3}\left(y^{2} x+y x y+x y^{2}\right)\end{array}$ & $\left.\begin{array}{ccc}1 & 0 & 0 \\
0 & 1 & 0 \\
1 & \frac{2}{3}+\gamma & 1\end{array}\right)$ \\
\hline
\end{tabular}


TABle 3. Continued.

\begin{tabular}{|c|c|c|}
\hline $\mathrm{TL}_{1}$ & $\begin{array}{l}x y z+y z x+z x y-(x z y+z y x \\
+y x z)-x^{3}\end{array}$ & $\left.\begin{array}{ccc}1 & 0 & 0 \\
0 & \gamma & 0 \\
0 & 0 & \gamma^{-1}\end{array}\right)$ \\
\hline $\mathrm{TL}_{2}$ & $\begin{array}{l}x y z+y z x+z x y-(x z y+z y x \\
+y x z)-x^{3}\end{array}$ & $\left.\begin{array}{lll}1 & 0 & 0 \\
\beta & 1 & 0 \\
0 & 1 & 1\end{array}\right)$ \\
\hline $\mathrm{TL}_{3}$ & $\begin{array}{l}x y z+y z x+z x y-(x z y+z y x \\
+y x z)-x^{3}\end{array}$ & $\begin{array}{ccc}1 & 0 & 0 \\
0 & -1 & 0 \\
0 & 1 & -1\end{array}$ \\
\hline $\mathrm{TL}_{4}$ & $\begin{array}{l}x y z+y z x+z x y-(x z y+z y x \\
+y x z)-x^{3}\end{array}$ & $\left.\begin{array}{lll}1 & 0 & 0 \\
0 & 1 & 0 \\
1 & 0 & 1\end{array}\right)$ \\
\hline
\end{tabular}

We remark how we find $\theta$ in Table 3. For most cases, we deduce $\theta$ by using a third root of the Nakayama automorphism $v_{A}$ of $A^{!}$, where $v_{A}$ ! is as in Table 1 . Otherwise, we find $\theta$ by using calculation directly (see Lemma 2.11 and Remark 3.2 (1)).

By Lemmas 2.11 and 2.16, Proposition 3.1, and Theorems 3.3 and 3.4, the following theorem immediately holds.

THEOREM 3.5. Any potential $w$ in Table 1 is a regular twisted superpotential.

REMARK 3.6. It turns out from Theorem 3.5 that the defining relations listed in [10, Theorem 3.1] are in fact those of three-dimensional quadratic AS-regular algebras (see also Remark 2.20).

By Theorem 3.4, for a three-dimensional AS-regular algebra $A$ except for Type EC, there exist a Calabi-Yau superpotential $w_{0}$ and $\theta \in \operatorname{Aut}\left(w_{0}\right)$ in Table 3 such that $A \cong$ $\mathcal{D}\left(\left(w_{0}\right)^{\theta}\right)$. Since $\theta \in \operatorname{Aut}\left(w_{0}\right)$, we have that $\mathcal{D}\left(\left(w_{0}\right)^{\theta}\right) \cong \mathcal{D}\left(w_{0}\right)^{\theta}$ by Lemma 2.10. Since $\mathcal{D}\left(w_{0}\right)$ is Calabi-Yau AS-regular, we have the following corollary.

COROLlARY 3.7. For any three-dimensional quadratic AS-regular algebra A except for Type EC, there exist a Calabi-Yau AS-regular algebra $S$ and $\theta \in$ Aut $S$ such that $A$ is isomorphic to $S^{\theta}$ as graded k-algebras.

\section{Geometric algebras of Type EC}

We say that a geometric algebra $A=\mathcal{A}(E, \sigma)$ is of Type EC if $E$ is an elliptic curve in $\mathbb{P}^{2}$. In this section we give a criterion when a geometric algebra of Type EC is a three-dimensional quadratic AS-regular algebra. 
4.1. Divisors on curves and Hesse forms. Let $E$ be a projective smooth curve over $k$. The Picard group of $E$, denoted by (Pic $\left.E,\left[O_{E}\right], \otimes\right)$, is the group of isomorphism classes of invertible sheaves on $E$ under the operation $\otimes$ (see [9, page 143]).

A divisor on $E$ is an element of the free abelian group

$$
\operatorname{Div} E:=\left\{\sum_{p \in E} n_{p} \cdot p \mid n_{p} \in \mathbb{Z}\right\}
$$

where only finitely many $n_{p}$ are different from zero. We write the group of divisors (Div $E, 0,+$ ) where 0 is the zero divisor, that is, $n_{p}=0$ for all $p \in E$. For any divisor $D \in$ Div $E$, there exists an invertible sheaf on $E$, denoted by $O_{E}(D)$, and the map $D \mapsto$ $O_{E}(D)$ gives a surjective homomorphism from (Div $E, 0,+$ ) to $\left(\mathrm{Pic} E,\left[O_{E}\right], \otimes\right)$ (see [9, Proposition II 6.13] and [9, Corollary II 6.16]), that is, for any [M] $\in \operatorname{Pic} E$ there exists a divisor $D \in \operatorname{Div} E$ such that $[\mathcal{M}]=\left[O_{E}(D)\right]$. The zero divisor 0 maps to the isomorphism class $\left[O_{E}\right] \in \mathrm{Pic} E$.

For $\sigma \in$ Aut $_{k} E$, we define a map $\tilde{\sigma}: \operatorname{Div} E \rightarrow \operatorname{Div} E$ by

$$
\tilde{\sigma}\left(\sum_{p \in E} n_{p} \cdot p\right)=\sum_{p \in E} n_{p} \cdot \sigma^{-1}(p) .
$$

This map $\tilde{\sigma}$ is a group automorphism of (Div $E, 0,+$ ). On the other hand, for $\sigma \in$ Aut $_{k} E$, the rule $\mathcal{M} \mapsto \sigma^{*} \mathcal{M}$, where $\mathcal{M}$ is an invertible sheaf on $E$, induces a group automorphism of the Picard group $\sigma^{*}:$ Pic $E \rightarrow$ Pic $E$. It follows from [9, II Ex. 6.8] that, if $D \in \operatorname{Div} E$, then

$$
\sigma^{*}\left(O_{E}(D)\right) \cong O_{E}(\tilde{\sigma} D) \text {. }
$$

Let $E$ be an elliptic curve in $\mathbb{P}^{2}$. It is well known that the $j$-invariant $j(E)$ classifies elliptic curves up to isomorphism; that is, two elliptic curves $E$ and $E^{\prime}$ in $\mathbb{P}^{2}$ are isomorphic if and only if $j(E)=j\left(E^{\prime}\right)$ (see [9, Theorem IV 4.1(b)]). For $p \in E$, we define $\operatorname{Aut}_{k}(E, p):=\left\{\sigma \in \operatorname{Aut}_{k} E \mid \sigma(p)=p\right\}$. It follows from [9, Corollary IV 4.7] that, for every point $p \in E$, $\operatorname{Aut}_{k}(E, p)$ becomes a cyclic group of order

$$
\left|\operatorname{Aut}_{k}(E, p)\right|= \begin{cases}2 & \text { if } j(E) \neq 0,12^{3}, \\ 6 & \text { if } j(E)=0, \\ 4 & \text { if } j(E)=12^{3} .\end{cases}
$$

For each point $o \in E$, we can define an addition $\oplus$ on $E$ so that $(E, o, \oplus)$ is an abelian group with the zero element $o$ and, for $p \in E$, the map $\sigma_{p}$ defined by $\sigma_{p}(q):=p \oplus q$ is a scheme automorphism of $E$, called the translation by a point $p$.

4.2. Type EC. Throughout this subsection for an elliptic curve $E$ in $\mathbb{P}^{2}$, we use a Hesse form $E=\mathcal{V}\left(x^{3}+y^{3}+z^{3}-3 \lambda x y z\right)$ where $\lambda \in k$ with $\lambda^{3} \neq 1$. The $j$-invariant of a Hesse form is given by the following formula (see [7, Proposition 2.16]):

$$
j(E)=\frac{27 \lambda^{3}\left(\lambda^{3}+8\right)^{3}}{\left(\lambda^{3}-1\right)^{3}} .
$$


We fix the group structure on $E$ with the zero element

$$
o_{E}:=(1:-1: 0) \in E .
$$

Every automorphism $\sigma \in \operatorname{Aut}_{k} E$ can be written as $\sigma=\sigma_{p} \tau^{i}$ where $p \in E, \tau$ is a generator of $\operatorname{Aut}_{k}\left(E, o_{E}\right)$ and $i \in \mathbb{Z}_{|\tau|}[10$, Proposition 4.5 and Theorem 4.6].

A point $p \in E$ is called 3-torsion if $p \oplus p \oplus p=o_{E}$. We set

$$
E[3]:=\left\{p \in E \mid p \oplus p \oplus p=o_{E}\right\} .
$$

For $p \in E$ and $i \in \mathbb{Z}, A=\mathcal{A}\left(E, \sigma_{p} \tau^{i}\right)$ is of Type EC if and only if $p \in E \backslash E[3]$ [10, Lemma 4.14].

The map $p \mapsto\left[O_{E}\left(p-o_{E}\right)\right]$ is an injective homomorphism from $\left(E, o_{E}, \oplus\right)$ to $\left(\operatorname{Pic} E,\left[O_{E}\right], \otimes\right)$ (see [9, Example IV 1.3.7]). For $p \in E$ and $n \in \mathbb{Z}$, we use the notation

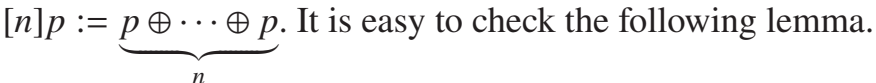

LEMMA 4.1. Let $\left(E, o_{E}, \oplus\right)$ be an elliptic curve in $\mathbb{P}^{2}, p \in E$ and $n \in \mathbb{Z}$. Then

$$
\left[O_{E}\left([n] p-o_{E}\right)\right]=\left[O_{E}\left(n\left(p-o_{E}\right)\right)\right] .
$$

Since the zero element $o_{E}=(1:-1: 0)$ is an inflection point of $E$, it follows that $\mathcal{L} \cong O_{E}\left(3 o_{E}\right)$ where $\mathcal{L}=\pi^{*}\left(O_{\mathbb{P}^{2}}(1)\right)$.

LeMma 4.2 cf. [12, Lemma 4.5]. Let $\pi: E \rightarrow \mathbb{P}^{2}$ be the embedding and $\mathcal{L}=\pi^{*} O_{\mathbb{P}^{2}}(1)$. Then an automorphism $\sigma \in$ Aut $_{k} E$ can be extended to an automorphism of $\mathbb{P}^{2}$ if and only if $\sigma^{*} \mathcal{L} \cong \mathcal{L}$.

A three-dimensional Sklyanin algebra is defined by $\mathcal{A}\left(E, \sigma_{p}\right)$ where a point

$$
p=(a: b: c) \in E \backslash E[3]
$$

and the defining relations are given as follows:

$$
\left\{\begin{array}{l}
a y z+b z y+c x^{2}, \\
a z x+b x z+c y^{2}, \\
a x y+b y x+c z^{2} .
\end{array}\right.
$$

A three-dimensional Sklyanin algebra $\mathcal{A}\left(E, \sigma_{p}\right)$ is a three-dimensional quadratic AS-regular algebra by [3, Section 1]. It follows from [10, Theorem 4.12 (1)] that $\sigma_{p} \tau^{i}$ is not extended to an automorphism of $\mathbb{P}^{2}$, so, by Lemma 4.2 ,

$$
\left(\sigma_{p} \tau^{i}\right)^{*} \mathcal{L} \neq \mathcal{L} \text {. }
$$

For a geometric algebra $A=\mathcal{A}(E, \sigma)$ of Type EC, we give a criterion when $A$ is AS-regular.

THEOREM 4.3. Let $A=\mathcal{A}(E, \sigma)$ be a geometric algebra of Type EC where $\sigma=\sigma_{p} \tau^{i}$, $p \in E \backslash E[3]$ and $i \in \mathbb{Z}_{|\tau|}$. Then the following are equivalent. 
(1) A is a three-dimensional quadratic AS-regular algebra.

(2) $p \ominus \tau^{i}(p) \in E[3]$.

(3) A is graded Morita equivalent to a three-dimensional Sklyanin algebra $\mathcal{A}\left(E, \sigma_{p}\right)$.

Proof. (1) $\Rightarrow$ (2). Assume that $A=\mathcal{A}(E, \sigma)$ is a three-dimensional quadratic AS-regular algebra. By Theorem 2.19, we have that

$$
\left(\sigma^{2}\right)^{*} \mathcal{L} \otimes_{O_{E}} \mathcal{L} \cong \sigma^{*} \mathcal{L} \otimes_{O_{E}} \sigma^{*} \mathcal{L} .
$$

Since $\mathcal{L} \cong O_{E}\left(3 o_{E}\right)$,

$$
\begin{aligned}
& \sigma^{*} \mathcal{L} \cong \sigma^{*}\left(O_{E}\left(3 o_{E}\right)\right) \cong O_{E}\left(\widetilde{\sigma}\left(3 o_{E}\right)\right)=O_{E}(3 q), \\
& \left(\sigma^{2}\right)^{*} \mathcal{L} \cong\left(\sigma^{2}\right)^{*}\left(O_{E}\left(3 o_{E}\right)\right) \cong O_{E}\left(\widetilde{\sigma^{2}}\left(3 o_{E}\right)\right)=O_{E}(3 r),
\end{aligned}
$$

where $q:=\sigma^{-1}\left(o_{E}\right)=\ominus \tau^{-i}(p)$ and $r:=\sigma^{-2}\left(o_{E}\right)=\sigma^{-1}(q)=q \oplus \tau^{-i}(q)$, so

$$
\begin{aligned}
& \left(\sigma^{2}\right)^{*} \mathcal{L} \otimes_{O_{E}} \mathcal{L} \cong O_{E}(3 r) \otimes_{O_{E}} O_{E}\left(3 o_{E}\right) \cong O_{E}\left(3\left(r+o_{E}\right)\right), \\
& \sigma^{*} \mathcal{L} \otimes_{O_{E}} \sigma^{*} \mathcal{L} \cong O_{E}(3 q) \otimes_{O_{E}} O_{E}(3 q) \cong O_{E}(6 q) .
\end{aligned}
$$

Therefore,

$$
\begin{aligned}
\left(\sigma^{2}\right)^{*} \mathcal{L} \otimes_{O_{E}} \mathcal{L} \cong \sigma^{*} \mathcal{L} \otimes_{O_{E}} \sigma^{*} \mathcal{L} & \Longrightarrow O_{E}\left(3\left(r+o_{E}\right)\right) \cong O_{E}(6 q) \\
& \Longrightarrow O_{E}\left(3\left(r-o_{E}\right)\right) \cong O_{E}\left(6\left(q-o_{E}\right)\right) \\
& \Longrightarrow O_{E}\left([3] r-o_{E}\right) \cong O_{E}\left([6] q-o_{E}\right) \\
& \Longrightarrow[3] r=[6] q \\
& \Longrightarrow[3]\left(q \ominus \tau^{-i}(q)\right)=o_{E} \\
& \Longrightarrow q \ominus \tau^{-i}(q) \in E[3] .
\end{aligned}
$$

Since $q=\ominus \tau^{-i}(p), q \ominus \tau^{-i}(q)=\ominus \tau^{-i}(p) \oplus \tau^{-2 i}(p)=\tau^{-2 i}\left(p \ominus \tau^{i}(p)\right)$. Hence we have $p \ominus \tau^{i}(p) \in E[3]$.

(2) $\Rightarrow$ (3). Assume that $p \ominus \tau^{i}(p) \in E[3]$. By [10, Theorem 4.20], $A=\mathcal{A}\left(E, \sigma_{p} \tau^{i}\right)$ and $\mathcal{A}\left(E, \sigma_{p}\right)$ are graded Morita equivalent.

(3) $\Rightarrow$ (1). Assume that $A=\mathcal{A}\left(E, \sigma_{p} \tau^{i}\right)$ is graded Morita equivalent to a three-dimensional Sklyanin algebra $\mathcal{A}\left(E, \sigma_{p}\right)$. By Lemma 2.5, $A$ is isomorphic to a twisted algebra of $\mathcal{A}\left(E, \sigma_{p}\right)$. Since being AS-regular is invariant under twisting systems by Lemma 2.6, a twisted algebra of $\mathcal{A}\left(E, \sigma_{p}\right)$ is a three-dimensional quadratic AS-regular algebra. Therefore, $A=\mathcal{A}\left(E, \sigma_{p} \tau^{i}\right)$ is a three-dimensional quadratic AS-regular algebra.

we are now ready to prove Theorem 1.1.

THEOREM 4.4. For every three-dimensional quadratic AS-regular algebra A, there exists a Calabi-Yau AS-regular algebra $S$ such that $A$ and $S$ are graded Morita equivalent. 
PROOF. By Lemma 2.5 and Corollary 3.7, the statement holds, except for Type EC.

For Type EC, let $A=\mathcal{A}\left(E, \sigma_{p}\right)=\mathcal{D}(w)$ be a three-dimensional Sklyanin algebra where $p=(a: b: c) \in E \backslash E[3]$ and

$$
w=a(x y z+y z x+z x y)+b(x z y+z y x+y x z)+c\left(x^{3}+y^{3}+z^{3}\right) .
$$

Since $w$ is a superpotential, by Lemma $2.15, \mathcal{D}(w)$ is Calabi-Yau AS-regular. By Theorem 4.3, the statement holds.

Corollary 3.7 tells us that, for a three-dimensional AS-regular algebra except for Type EC, there exist a Calabi-Yau AS-regular algebra $S$ and $\theta \in$ Aut $S$ such that $A$ is isomorphic to $S^{\theta}$ as graded $k$-algebras. We prove this by using Theorem 3.4, that is, for a potential $w$ in Theorem 3.1, there exist a superpotential $w_{0}$ in Theorem 3.3 and $\theta \in$ Aut $\left(w_{0}\right)$ such that $\mathcal{D}(w) \cong \mathcal{D}\left(\left(w_{0}\right)^{\theta}\right)$. On the other hand, it follows from [10, Theorem 4.9] that, for a potential $w$ of a geometric algebra of Type EC, there exist a Calabi-Yau superpotential $w_{0}$ and $\theta \in \mathrm{GL}(V)$ induced by $\tau^{i} \in$ Aut $_{k} E$ such that $w=\left(w_{0}\right)^{\theta}$. But this $\theta$ is not necessarily in Aut $\left(w_{0}\right)$, so $w=\left(w_{0}\right)^{\theta}$ need not be a twisted superpotential or regular (see Example 4.6). This means that we do not know whether or not Theorem 3.4 holds for Type EC. So, we need to divide the proof of Theorem 1.1 into two cases of non-Type EC and Type EC.

EXAMPLE 4.5. Let $E=\mathcal{V}\left(x^{3}+y^{3}+z^{3}-3 \lambda x y z\right)$ be an elliptic curve in $\mathbb{P}^{2}$ with $j(E) \neq$ $0,12^{3}$ and $A=\mathcal{A}\left(E, \sigma_{p} \tau\right)$ where $p \in E \backslash E[3]$. In this case, we have that $\tau(p)=\ominus p$. By Theorem 4.3, $A$ is a three-dimensional quadratic AS-regular algebra if and only if [2] $p \in E[3]$, that is, $p \in E[6]$ where $E[6]:=\left\{q \in E \mid[6] q=o_{E}\right\}$.

EXAMPLE 4.6. In general, it is not true that if $w$ is a regular superpotential and $\theta \in$ $\mathrm{GL}(V)$, then the MS twist $w^{\theta}$ is regular. Let

$$
E=\mathcal{V}\left(x^{3}+y^{3}+z^{3}-3 \lambda x y z\right)
$$

be an elliptic curve with $j(E) \neq 0,12^{3}$ and $A=\mathcal{A}\left(E, \sigma_{p} \tau\right)$ where a point

$$
p=(a: b: c) \in E \backslash E[6]
$$

By Theorem 4.3, $A$ is not AS-regular. By [10, Theorem 4.6] and [10, Theorem 4.9], we have that $A=\mathcal{A}\left(E, \sigma_{p} \tau\right)=\mathcal{D}\left(w^{\theta}\right)$ where $w=a(x y z+y z x+z x y)+b(x z y+y x z+$ $z y x)+c\left(x^{3}+y^{3}+z^{3}\right)$ and

$$
\theta=\left(\begin{array}{ccc}
0 & 1 & 0 \\
1 & 0 & 0 \\
0 & 0 & 1
\end{array}\right) \in \mathrm{GL}_{3}(k)
$$

Note that if $a \neq b$, then $\theta \notin \operatorname{Aut}(w)$. Since $A$ is not a three-dimensional quadratic AS-regular algebra, $w^{\theta}$ is not regular. 


\section{Acknowledgements}

The authors thank the referee for helpful comments in improving the paper. They are grateful to Professor Izuru Mori for his support and helpful discussions. Also, they would like to thank Professor Kenta Ueyama for his helpful comments. Moreover, they appreciate Shinichi Hasegawa and Kosuke Shima for helping to build and to check Table 1 in Proposition 3.1. For Remark 3.2 (2), the authors thank Professor Andrew Conner and Professor Peter Goetz for informing them of a typo for the relation of Type $\mathrm{TL}_{4}$.

\section{References}

[1] M. Artin and W. Schelter, 'Graded algebras of global dimension 3', Adv. Math. 66 (1987), 171-216.

[2] M. Artin, J. Tate and M. Van den Bergh, 'Some algebras associated to automorphisms of elliptic curves', in: The Grothendieck Festschrift, Vol. 1, Progress in Mathematics, 86 (Birkhäuser , Basel, 1990), 33-85.

[3] M. Artin and J. J. Zhang, 'Noncommutative projective schemes', Adv. Math. 109(2) (1994), 228-287.

[4] R. Bocklandt, 'Graded Calabi Yau algebras of dimension 3', J. Pure Appl. Algebra 212(1) (2008), $14-32$.

[5] R. Bocklandt, T. Schedler and M. Wemyss, 'Superpotentials and higher order derivations', J. Pure Appl. Algebra 214 (2010), 1501-1522.

[6] M. Dubois-Violette, 'Multilinear forms and graded algebras', J. Algebra 317 (2007), 198-225.

[7] H. R. Frium, 'The group law on elliptic curves on Hesse form', in: Finite Fields with Applications to Coding Theory, Cryptography and Related Areas (Oaxaca, 2001) (Springer, Berlin, 2002), 123-151.

[8] V. Ginzburg, 'Calabi-Yau algebras', Preprint, 2007, arXiv:0612139.

[9] R. Hartshorne, Algebraic Geometry, Graduate Texts in Mathematics, 52 (Springer-Verlag, New York, 1977).

[10] A. Itaba and M. Matsuno, 'Defining relations of 3-dimensional quadratic AS-regular algebras', Math. J. Okayama Univ. 63 (2021), 61-86.

[11] N. Iyudu and S. Shkarin, 'Classifcation of quadratic and cubic PBW algebras on three generators', Preprint, 2018, arXiv:1806.06844.

[12] I. Mori, 'Non commutative projective schemes and point schemes', in: Algebras, Rings and Their Representations (World Scientific, Hackensack, NJ, 2006), 215-239.

[13] I. Mori and S. P. Smith, ' $m$-Koszul Artin-Schelter regular algebras', J. Algebra 446 (2016), 373-399.

[14] I. Mori and S. P. Smith, 'The classification of 3-Calabi-Yau algebras with 3 generators and 3 quadratic relations', Math. Z. 287(1-2) (2017), 215-241.

[15] I. Mori and K. Ueyama, 'Graded Morita equivalences for geometric AS-regular algebras', Glasg. Math. J. 55(2) (2013), 241-257.

[16] M. Reyes, D. Rogalski and J. J. Zhang, 'Skew Calabi-Yau algebras and homological identities', Adv. Math. 264 (2014), 308-354.

[17] S. P. Smith, 'Some finite dimensional algebras related to elliptic curves', in: Representation Theory of Algebras and Related Topics (Mexico City, 1994), CMS Conference Proceedings, 19 (American Mathematical Society, Providence, RI, 1996), 315-348.

[18] K. Ueyama, '3-dimensional cubic Calabi-Yau algebras and superpotentials', Proc. 49th Symp. Ring Theory Represent. Theory (Symposium on Ring Theory and Representation Theory Organizing Committee, Shimane, 2017), 155-160.

[19] J. J. Zhang, 'Twisted graded algebras and equivalences of graded categories', Proc. Lond. Math. Soc. 72 (1996), 281-311. 
AYAKO ITABA, Department of Mathematics, Faculty of Science, Tokyo University of Science, 1-3 Kagurazaka, Shinjyuku-ku, Tokyo 162-8601, Japan

and

Institute of Arts and Sciences, Katsushika Division,

Tokyo University of Science, 6-3-1 Niijjuku, Katsushika-ku, Tokyo 125-8585, Japan

e-mail: itaba@rs.tus.ac.jp

MASAKI MATSUNO, Graduate School of Science and Technology, Shizuoka University, Ohya 836,

Shizuoka 422-8529, Japan

e-mail: matsuno.masaki.14@shizuoka.ac.jp 